\title{
Neurochemical and electrophysiological diagnosis of reversible neurotoxicity in earthworms exposed to sublethal concentrations of CL-20
}

\author{
Ping Gong • Niladri Basu • Anton M. Scheuhammer • \\ Edward J. Perkins
}

Received: 3 October 2008 / Accepted: 13 February 2009/Published online: 10 March 2009

(C) The Author(s) 2009. This article is published with open access at Springerlink.com

\begin{abstract}
Background, aim, and scope Hexanitrohexaazaisowurtzitane (CL-20) is a relatively new energetic compound sharing some degree of structural similarity with hexahydro-1,3,5-trinitro1,3,5-triazine (RDX), a known neurotoxic compound. Previously, we demonstrated using a noninvasive electrophysiological technique that CL-20 was a more potent neurotoxicant than RDX to the earthworm Eisenia fetida. In the present study, we investigated the effect of CL-20 exposure and subsequent recovery on muscarinic acetylcholine receptors (mAChRs) to further define the mechanism of reversible neurotoxicity of CL-20 in E. fetida.

Materials and methods We used a noninvasive electrophysiological technique to evaluate neurotoxicity in CL-20-
\end{abstract}

Responsible editor: Kerstin Hund-Rinke

P. Gong $(\bowtie)$

SpecPro Inc.,

3909 Halls Ferry Road,

Vicksburg, MS 39180, USA

e-mail: ping.gong@us.army.mil

N. Basu

Department of Environmental Health Sciences,

University of Michigan,

Ann Arbor, MI 48109, USA

\section{A. M. Scheuhammer}

National Wildlife Research Center, Canadian Wildlife Service,

Environment Canada,

Ottawa, ON K1A 0H3, Canada

\section{E. J. Perkins}

Environmental Laboratory,

US Army Engineer Research and Development Center,

3909 Halls Ferry Road,

Vicksburg, MS 39180, USA treated worms, and then measured how such exposures altered levels of whole-body $\mathrm{mAChR}$ in the same animals. Results and discussion A good correlation exists between these two types of endpoints. Effect on mAChR levels was most prominent at day 6 of exposure. After 7 days of recovery, both conduction velocity and $\mathrm{mAChR}$ were significantly restored. Our results show that sublethal concentrations of CL-20 significantly reduced mAChR levels in a concentration- and duration-dependent manner, which was accompanied with significant decreases in the conduction velocity of the medial and lateral giant nerve fibers. After 7-day post exposure recovery, worms restored both neurochemical (mAChR) and neurophysiological (conduction velocity) endpoints that were reduced during 6-day exposures to CL-20 concentrations from 0.02 to $0.22 \mu \mathrm{g} / \mathrm{cm}^{2}$.

Conclusions and perspectives Our findings support the idea that CL-20 induced neurotoxic effects are reversible, and suggest that CL-20 neurotoxicity may be mediated through the cholinergic system. Future studies will investigate other neurotransmission systems such as GABA, glutamate, and monoamine. Ion channels in the nerve membrane should be examined to further define the precise mechanisms underlying CL-20 neurotoxicity.

Keywords Conduction velocity - Earthworm (Eisenia fetida) - Electrophysiological recording · Giant nerve fiber . Hexanitrohexaazaisowurtzitane (CL-20) · Muscarinic acetylcholine receptor $(\mathrm{mAChR}) \cdot$ Reversible neurotoxicity

\section{Background, aim, and scope}

The use of earthworms for soil ecotoxicological risk assessment has advanced significantly over the past few 
decades. As many environmental contaminants possess neurotoxic properties, there exists a growing interest in using earthworms as neurotoxicity test organisms for environmental risk assessment and as potential surrogates for evaluating impacts of neurotoxic compounds on humans (Davoli et al. 2002; Mizutani et al. 2003; Costa et al. 2008).

Neurotoxicity can be defined as any adverse effect on the central or peripheral nervous system caused by chemical, biological or physical agents (Costa et al. 2008). Upon high, acute exposures, neurotoxicity can be manifested as severe clinical signs and structural disorders (e.g., brain lesions, neurobehavioral changes, lethality). More often, organisms are chronically exposed to lowlevels of neurotoxic substances. Over time, such exposures can result in subtle, sub-clinical effects that include, for example alterations in neurochemical function and nerve conduction. The ability to diagnose these early neurological effects not only warns of imminent neurotoxic damage but also provides important information as to cellular mechanisms of action (Manzo et al. 1996).

CL-20 (2,4,6,8,10,12-hexanitro-2,4,6,8,10,12-hexaazaisowurtzitane) is a relatively new energetic compound sharing some degree of structural similarity with hexahydro-1,3,5-trinitro-1,3,5-triazine (RDX), a widely used explosive with known neurotoxic properties in both humans and animals (Davies et al. 2007; Johnson et al. 2007). Both CL-20 and RDX are recalcitrant to degradation resulting in persistence in the environment (Crocker et al. 2005) and posing potential environmental risks to soil invertebrates as soil is the main sink. Recently, we have demonstrated that earthworms (Eisenia fetida) exposed to CL-20 or RDX exhibited typical neurophysiological symptoms (e.g., rigidity, coiling and ataxia) and that sublethal concentrations of either compound caused reversible neurotoxicity (Gong et al. 2007). These results raised further questions regarding specific sites of action and molecular targets within the worm's central and peripheral nervous systems. A preliminary neurochemical investigation indicated that 8-day exposure to RDX (1.07 or $5.35 \mu \mathrm{g} / \mathrm{cm}^{2}$ filter paper) significantly affected whole-body, muscarinic acetylcholine receptor (mAChR) levels in E. fetida, and these responses were accompanied by a significant decrease in the relative conduction velocity of both medial (MGF) and lateral (LGF) giant nerve fibers (Gong et al. 2006). However, no significant effects of RDX were observed on other neurochemical parameters, including monoamine oxidase, acetylcholine, and cholinesterase in these same worms (Gong et al. 2006).

Given the above results and the structural similarity characterized by the $\mathrm{CH}-\mathrm{N}-\mathrm{NO}_{2}$ bonds present in both RDX and CL-20, we hypothesize that CL-20 affects the $\mathrm{mAChR}$. Although the mAChR has crucial functions within the central nervous system, emerging evidence suggests that the $\mathrm{mAChR}$ also plays an important role in neuromuscular junctions of the peripheral nervous system (Santafe et al. 2003; Rand 2007). In the present study, we used a noninvasive electrophysiological technique to evaluate neurotoxicity in CL-20-treated worms, and then measured how such exposures altered levels of whole-body mAChR in the same animals. Our ultimate long-term goal is to elucidate the mechanisms underlying the reversible neurotoxicity of sublethal CL-20 concentrations in earthworms (Gong et al. 2007).

\section{Materials and methods}

\subsection{Earthworm culture and exposure}

Earthworms (E. fetida) were maintained in a continuous culture from stocks obtained from Carolina Biological Supply Company (Burlington, NC, USA). Worms were kept at 22 to $25^{\circ} \mathrm{C}$ in moistened sphagnum peat with calcium carbonate added to adjust the $\mathrm{pH}$ to 6.5 to 7.5 and moisture content adjusted to $50 \%$ and were fed ad libitum on a diet of Magic Worm Food (Carolina Biological Supply). Adult earthworms (0.3 0.6 g with clitellum) were purged overnight on moistened filter paper prior to testing. Exposures were performed in 95-ml capped glass vials via contact with moistened filter paper (Gong et al. 2007). One milliliter of CL-20 solution in methanol was spread on the filter paper $\left(115 \mathrm{~cm}^{2}\right)$ and methanol was allowed to evaporate before moistening the filter paper with $2 \mathrm{ml}$ of de-ionized water. The concentration of CL-20 in stock solutions ( $\mu \mathrm{g}$ CL-20/ml of methanol) was confirmed by HPLC analysis (Gong et al. 2008) and was converted into $\mu \mathrm{g} / \mathrm{cm}^{2}$ to express concentration of exposure. The $\varepsilon$-CL-20 (99.3\%) was synthesized by ATK Thiokol Propulsion (China Lake, UT, USA). Gas chromatographic-grade methanol was purchased from Burdick and Jackson (Muskegon, MI, USA).

\subsection{Experimental design}

Four sets of experiments were carried out with duration of 1, 3, 6, and 13 days, respectively. Throughout all the experiments, each worm was housed individually in a vial. Only worms in the fourth experiment were removed and allowed to recover on clean filter paper for 7 days after a 6day exposure. Noninvasive electrophysiological measurements were repeatedly performed on day 0 (after purging but before exposure), 1, 3, 6, 7, 10 and 13 before the worms were sacrificed on day 1, 3, 6 and 13, respectively in the four experiments. Levels of muscarinic acetylcholine receptor $(\mathrm{mAChR})$ were determined only at the termination 
of each experiment when the worms were sacrificed by snap-freezing in liquid nitrogen. Four concentrations of CL20 were tested in each experiment, except for the second experiment where only three CL-20 concentrations were tested. Ten worms were used per treatment. The exposure concentration ( 0.02 to $0.87 \mu \mathrm{g} \mathrm{CL}-20 / \mathrm{cm}^{2}$ depending on the exposure length) was carefully selected to avoid lethality based on results from our previous studies (Gong et al. 2007).

\subsection{Noninvasive electrophysiological recording}

Conduction velocities of worm MGF and LGF were measured as previously described (Gong et al. 2007) immediately before exposure and at each interval of exposure and recovery. Absolute velocity $(\mathrm{m} / \mathrm{s})$ was calculated by dividing conduction distance between electrode pairs $(10 \mathrm{~mm})$ by conduction time. Relative conduction velocity, i.e., the absolute conduction velocity measured after exposure or recovery relative to that measured before exposure, was used as assessment endpoint because absolute conduction velocity varied from one worm to another, even in the same treatment groups.

\subsection{Muscarinic acetylcholine receptor (mAChR) binding assay}

The level of whole-body mAChR was analyzed in homogenized worms as described in Basu et al. (2006) with slight modifications. All worm samples were homogenized for $30 \mathrm{~s}$ in cold $\mathrm{Na} / \mathrm{K}$ buffer $\left(50 \mathrm{mM} \mathrm{NaH} \mathrm{PO}_{4}\right.$, $5 \mathrm{mM} \mathrm{KCl}, 120 \mathrm{mM} \mathrm{NaCl}, \mathrm{pH}$ 7.4). Cellular membranes were isolated by centrifuging the homogenate at $32,500 \times \mathrm{g}$ for $15 \mathrm{~min}$ at $4^{\circ} \mathrm{C}$. The resulting pellet was washed twice under the same conditions, and the final pellet was resuspended in $\mathrm{Na} / \mathrm{K}$ buffer. Protein concentration was determined using the Bradford protocol. Binding to the $\mathrm{mACh}$ receptor was performed in a 96-well $1.0 \mu \mathrm{M} \mathrm{GF} / \mathrm{B}$ glass filter system (Millipore, Boston, MA, USA) (Stamler et al. 2005). Approximately $20 \mu \mathrm{g}$ of membrane preparation in $\mathrm{Na} / \mathrm{K}$ buffer was incubated with $1 \mathrm{nM}[3 \mathrm{H}]$-quinuclidinyl benzilate $([3 \mathrm{H}]-\mathrm{QNB}$; NEN/Perkin Elmer, Waltham, MA, USA). All binding assays were carried out under gentle agitation for $60 \mathrm{~min}$, and reactions were terminated by vacuum filtration. The filters were rinsed three times with buffer and then allowed to soak for $96 \mathrm{~h}$ in $25 \mu \mathrm{L}$ of OptiPhase Supermix Cocktail (Perkin Elmer). Radioactivity retained by the filter was quantified by liquid scintillation counting in a microplate detector (Wallac Microbeta, Perkin Elmer). Specific binding to the receptors was defined as the difference in radioligand bound in the presence and absence of $100 \mu \mathrm{M}$ atropine sulfate.

\subsection{Statistical data analysis}

The electrophysiological data were analyzed using a paired Student's $t$ test by comparing the absolute MGF/LGF conduction velocity measured at different time points with that measured before exposure (day 0) in the same worms within the same treatment group. One-way analysis of variance (ANOVA) was used to determine the effects of CL-20 on mAChR levels. When a significant difference was found, a post-hoc test was performed with Tukey's HSD (honestly significant difference). The probability $(P)$ of type-1 or false positive error was derived from ANOVA, whereas the power $(B)$ of the statistical test was estimated from $B=1-\beta$ with $\beta$ being the type- 2 or false negative error probability and $\alpha$ being set at 0.05 . All the statistical analysis was performed using SigmaStat (v 3.1, Point Richmond, CA, USA).

\section{Results and discussion}

CL-20 exposure reduced both conduction velocity and whole-body mAChR levels in a concentration- and duration-dependent manner (Fig. 1). Although conduction velocity was recorded repeatedly, only results from the final measurement were shown to facilitate comparison with $\mathrm{mAChR}$ data obtained from single-time determinations (see Fig. 1). A good correlation exists between these two types of endpoints. Effect on mAChR levels was most prominent at day 6 of exposure (see Fig. 1(f)). After a 7-day recovery, both conduction velocity and $\mathrm{mAChR}$ were significantly restored (see Fig. 1(g,h)). However, it should be noted that relative conduction velocity is a more sensitive endpoint than mAChR, and this can be partly attributed to different statistical methods used. One-way ANOVA used for mAChR has less statistical power than the paired $t$ test for conduction velocity because ANOVA does not require pairing of collected data.

There was a sharp decrease in the whole-body levels of $\mathrm{mAChR}$ reflected by the $[3 \mathrm{H}]-\mathrm{QNB}$ binding activity in control worms from day 1 (mean \pm standard deviation= $101.9 \pm 27.0 \mathrm{fmol} / \mathrm{mg})$ to day $3(19.2 \pm 3.2 \mathrm{fmol} / \mathrm{mg}$; see Fig. 1(b, d)). It should be noted that there were no technical or experimental changes in the study over this time period, and this decrease may be due to the cessation of foraging activity and gut digestive movement as food supply was cut off on day 0 (You et al. 2006). However, this hypothesis requires further investigation. Although statistically significant $(\alpha=0.05)$ decreases in $\mathrm{mAChR}$ were measured in worms exposed to CL-20 for 1 day (see Fig. 1(b)) and 3 days (see Fig. 1(d)), the power associated with these changes (particularly in 3-day-exposed worms) was weak as the calculated $\mathrm{B}$ values were below the acceptable 
Fig. 1 Reversible effects of sublethal CL-20 concentrations on muscarinic acetylcholine receptor ( $\mathrm{mAChR}$ ) and conduction velocity of medial (MGF) and lateral (LGF) giant fiber. Data are presented as mean (bar) and standard error (error bar) with $n=10$. Statistical significance is indicated by an asterisk ' $*$ ' at $\alpha=0.05$. Endpoints were determined in worms exposed with or without recovery for 1 (a and $\mathbf{b}$ ), 3 (c and d), 6 (e and f), and 13 days ( $\mathbf{g}$ and $\mathbf{h})$ in four separate experiments. MGF and LGF were measured repeated but only the last measurement results $(\mathbf{a}, \mathbf{c}, \mathbf{e}$, and $\mathbf{g})$ are shown in parallel with $\mathrm{mAChR}$ results $(\mathbf{b}, \mathbf{d}, \mathbf{f}$, and $\mathbf{h})$. See "Materials and methods" for details on statistical data analysis
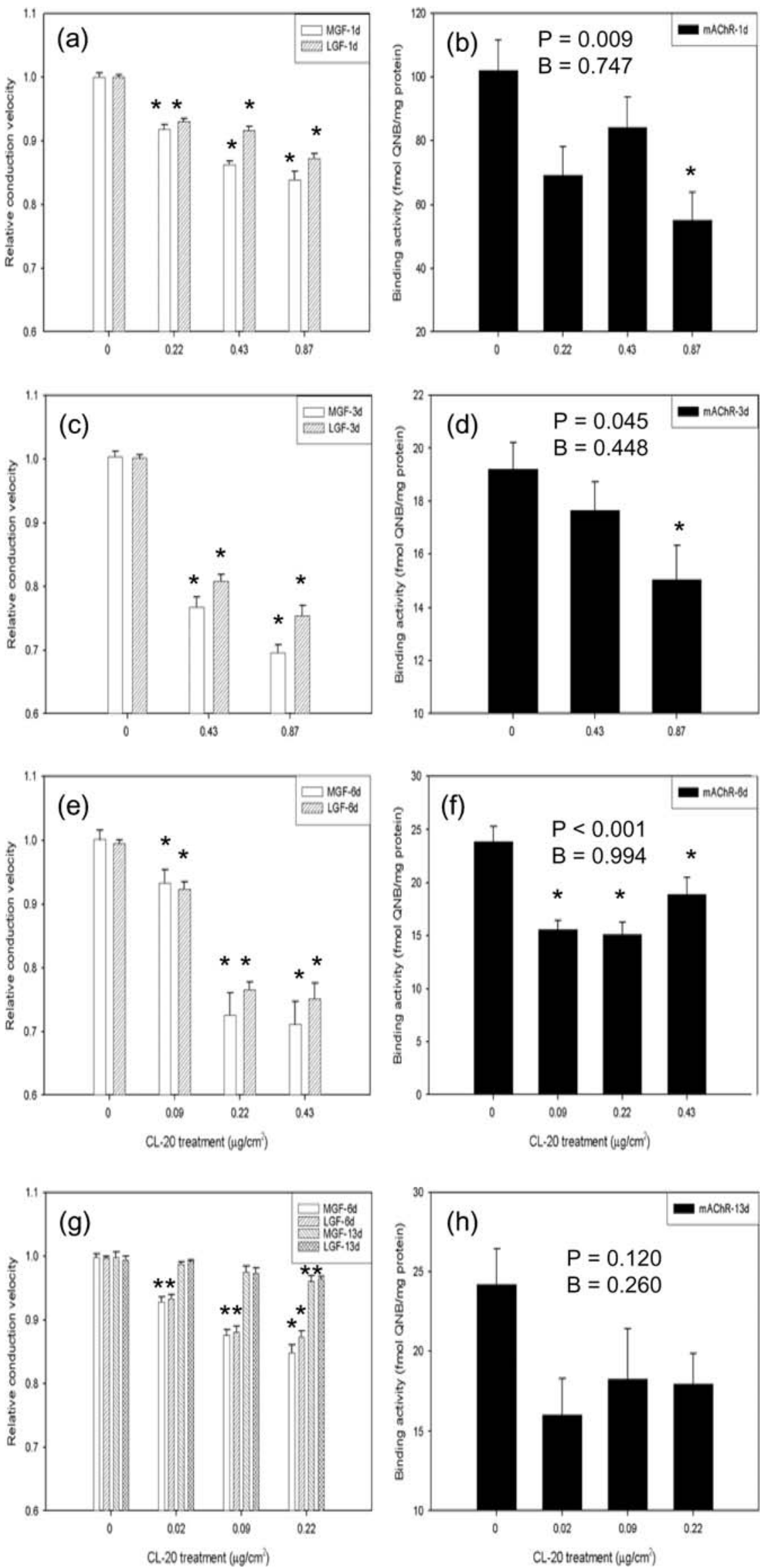
threshold of 0.8 . This may be partly attributed to neurological plasticity and inter-worm differences in their adaptive neurological response following relatively shortterm exposure to a toxicant like CL-20 (Bargmann 2006). While [3H]-QNB can label and enumerate the global population of mAChRs, it is well established that mAChRs consist of several different isoforms in both vertebrates and invertebrates as a result of alternative splicing, can exist at pre-synaptic and post-synaptic sites, and are found in both the central and peripheral nervous systems (Trimmer 1995; Wess 1996; Santafe et al. 2003; Rand 2007). Furthermore, a putative acetylcholine receptor with a mixed pharmacological property (i.e., binds to both nicotinic and muscarinic drugs) has been identified in invertebrates (Eldefrawi and Eldefrawi 1983; Trimmer 1995). It should be stressed that the $\mathrm{mAChR}$ data we present here represents the total amount of the mAChR and mixed nicotinic-muscarinic receptors in the whole body of earthworms. Future studies should differentiate the mAChR responses among the different receptor isoforms in both the central and peripheral nervous systems.

The strongest statistical results were obtained in worms following 6-day exposure when compared to animals exposed for 1 or 3 days (see Fig. 1(b, d, f)), indicating that longer-term exposure to CL-20 may have exceeded the adaptive capability of these worms. However, after a 7-day recovery period following a 6-day exposure period (see Fig. 1(h)), no statistically significant differences in mAChR levels were observed, suggesting that to a certain degree, the earthworms were capable of restoring cholinergic function. Recovery of these neurochemical responses were supported by the neurophysiological work that also showed a recovery response (see Fig. 1 (a, c, e, g)) (Gong et al. 2007).

\section{Conclusions and perspectives}

Multiple lines of evidence (i.e., behavioral observation (Gong et al. 2007), neurobiochemical and electrophysiological measurements) from our work strongly suggest that CL-20 has neurotoxic properties. Specifically, here we show that CL-20 can affect the mAChR in earthworms. However, this does not exclude the possibility that CL-20 may have other neurological targets. Many pesticides acting on neurotransmitter receptors (e.g., organophosphorus and carbamate insecticides) also inhibit cholinesterase (Eldefrawi and Eldefrawi 1983; Edwards and Fisher 1991), sometimes in a reversible manner (Aamodt et al. 2007). In addition, other neurotransmission systems such as GABA, glutamate, and monoamine (Barna et al. 2001; Csoknya et al. 2003) and ion channels in the nerve membrane (Ray and Fry 2006) should be examined to further define the precise mechanisms underlying CL-20 neurotoxicity.
Acknowledgments This study was funded by the U.S. Army Environmental Quality Technology Basic Research Program. Permission was granted by the Chief of Engineers to publish this information. We thank Willie Brown and Robert Boyd for their technical assistance.

Open Access This article is distributed under the terms of the Creative Commons Attribution Noncommercial License which permits any noncommercial use, distribution, and reproduction in any medium, provided the original author(s) and source are credited.

\section{References}

Aamodt S, Konestabo HS, Sverdrup LE, Gudbrandsen M, Reinecke SA, Reinecke AJ, Stenersen J (2007) Recovery of cholinesterase activity in the earthworm Eisenia fetida Savigny following exposure to chlorpyrifos. Environ Toxicol Chem 26:1963-1967

Bargmann CI (2006) Chemosensation in C. elegans. In: The $C$. elegans Research Community (ed), WormBook (http://www. wormbook.org), pp. 1-29.

Barna J, Csoknya M, Lazar Z, Bartho L, Hamori J, Elekes K (2001) Distribution and action of some putative neurotransmitters in the stomatogastric nervous system of the earthworm, Eisenia fetida (Oligochaeta, Annelida). J Neurocytol 30:313-325

Basu N, Scheuhammer AM, Rouvinen-Watt K, Grochowina N, Klenavic K, Evans RD, Chan HM (2006) Methylmercury impairs components of the cholinergic system in captive mink (Mustela vison). Toxicol Sci 91:202-209

Costa LG, Giordano G, Guizzetti M, Vitalone A (2008) Neurotoxicity of pesticides: a brief review. Front Biosci 13:1240-1249

Crocker FH, Thompson KT, Szecsody JE, Fredrickson HL (2005) Biotic and abiotic degradation of CL-20 and RDX in soils. J Environ Qual 34:2208-2216

Csoknya M, Barna J, Hiripi L, Hamori J, Elekes K (2003) Reorganization of monoaminergic systems in the earthworm, Eisenia fetida, following brain extirpation. J Exp Zoolog A Comp Exp Biol 296:18-29

Davies JO, Roberts DM, Hittarage A, Buckley NA (2007) Oral C-4 plastic explosive in humans - a case series. Clin Toxicol (Phila) 45:454-457

Davoli C, Marconi A, Serafino A, Iannoni C, Marcheggiano A, Ravagnan G (2002) Expression of nerve growth factor-like polypeptides and immunoreactivity related to the two types of neurotrophin receptors in earthworm tissues. Cell Mol Life Sci 59:527-539

Edwards CA, Fisher SW (1991) The use of cholinesterase measurement in assessing the impacts of pesticides on terrestrial and aquatic invertebrates. In: Mineau P (ed) Cholinesterase-inhibiting insecticides. Elsevier, Amsterdam, The Netherlands, pp 255-275

Eldefrawi ME, Eldefrawi AT (1983) Neurotransmitter receptors as targets for pesticides. J Environ Sci Health B 18:65-88

Gong P, Basu N, Perkins EJ (2006) Neurochemical and electrophysiological diagnosis of RDX and CL-20 neutotoxicity in the earthworm Eisenia fetida. In: Abstract Book of SETAC North America 27th Annual Meeting, Montreal, QC, Canada, November 5-9, 2006, p. 363

Gong P, Inouye LS, Perkins EJ (2007) Comparative neurotoxicity of two energetic compounds, hexanitrohexaazaisowurtzitane and hexahydro-1, 3, 5-trinitro-1, 3, 5-triazine, in the earthworm Eisenia fetida. Environ Toxicol Chem 26:954-959

Gong P, Escalon BL, Hayes CA, Perkins EJ (2008) Uptake of hexanitrohexaazaisowurtzitane (CL-20) by the earthworm Eisenia fetida through dermal contact. Sci Total Environ 390:295-299

Johnson MS, Quinn MJ, Bazar MA, Gust KA, Escalon BL, Perkins EJ (2007) Subacute toxicity of oral 2, 6-dinitrotoluene and 1, 3, 5- 
trinitro-1, 3, 5-triazine (RDX) exposure to the northern bobwhite (Colinus virginianus). Environ Toxicol Chem 26:1481-1487

Manzo L, Artigas F, Martinez E, Mutti A, Bergamaschi E, Nicotera P, Tonini M, Candura SM, Ray DE, Costa LG (1996) Biochemical markers of neurotoxicity: a review of mechanistic studies and applications. Hum Exp Toxicol 15(Suppl 1):S20-S35

Mizutani K, Shimoi T, Kitamura Y, Ogawa H, Oka K (2003) Identification of two types of synaptic activity in the earthworm nervous system during locomotion. Neurosci 121:473-478

Rand JB (2007) Acetylcholine. In: The C. elegans Research Community (ed), WormBook (http://www.wormbook.org), pp. $1-21$

Ray DE, Fry JR (2006) A reassessment of the neurotoxicity of pyrethroid insecticides. Pharmacol Ther 111:174-193
Santafe MM, Salon I, Garcia N, Lanuza MA, Uchitel OD, Tomas J (2003) Modulation of ACh release by presynaptic muscarinic autoreceptors in the neuromuscular junction of the newborn and adult rat. Eur J Neurosci 17:119-127

Stamler CJ, Basu N, Man CH (2005) Biochemical markers of neurotoxicity in wildlife and human populations: considerations for method development. J Toxicol Environ Health A 68:1413-1429

Trimmer BA (1995) Current excitement from insect muscarinic receptors. Trends Neurosci 18:104-111

Wess J (1996) Molecular biology of muscarinic acetylcholine receptors. Crit Rev Neurobiol 10:69-99

You YJ, Kim J, Cobb M, Avery L (2006) Starvation activates MAP kinase through the muscarinic acetylcholine pathway in Caenorhabditis elegans pharynx. Cell Metab 3:237-245 